\title{
Einfluss von natürlichen Lithiumsalzvorkommen auf die Suizidmortalität in Chile 2000-2009: Eine geographische Analyse
}

\author{
Daniel König (iD · Josef Baumgartner · Victor Blüml · Andrés Heerlein · Carlos Téllez • Nicole Baus · \\ Nestor D. Kapusta
}

Eingegangen: 29. Januar 2017 / Angenommen: 28. Februar 2017 / Online publiziert: 29. März 2017

(c) Der/die Autor(en) 2017. Dieser Artikel ist eine Open-Access-Publikation.

\begin{abstract}
Zusammenfassung
Grundlagen Es gibt zunehmende Evidenz für einen protektiven Effekt natürlicher Lithiumsalzvorkommen im Trinkwasser auf die Suizidraten der betroffenen Bevölkerung. Ziel der Studie war es zu untersuchen, ob die lithiumreiche Atacama-Region in Chile im Vergleich zu anderen Regionen mit einer niedrigeren Suizidmortalität assoziiert ist.

Methodik Suizidstatistiken wurden vom chilenischen Gesundheitsministerium erhoben. Sozioökonomische Variablen (Arbeitslosenrate, medianes Haushaltseinkommen, Grad der Urbanisierung, Anteil der indigenen Bevölkerung) aller chilenischen Regionen wurden vom nationalen Statistikinstitut angefordert. Wir be-
\end{abstract}

\section{König · J. Baumgartner}

Klinische Abteilung für Sozialpsychiatrie, Univ.-Klinik für Psychiatrie und Psychotherapie, Medizinische Universität Wien, 1090 Wien, Österreich

\section{König}

daniel.koenig@meduniwien.ac.at

J. Baumgartner

josef.baumgartner@meduniwien.ac.at

V. Blüml $•$ N. Baus $\cdot$ N. D. Kapusta, MD, PD $(\varangle)$

Suizidforschungsgruppe, Univ.-Klinik für Psychiatrie und Psychoanalyse, Medizinische Universität Wien, 1090 Wien, Österreich

nestor.kapusta@meduniwien.ac.at

V. Blüml

viktor.blueml@meduniwien.ac.at

N. Baus

nicole.baus@web.de

\section{A. Heerlein · C. Téllez}

Klinik für Psychiatrie, Facultad de Medicina Clínica Alemana - Universidad del Desarrollo, Las Condes, Santiago de Chile, Chile

aheerlein@alemana.cl rechneten die jährliche Suizidraten pro 100.000 jeder Region in den Jahren 2000-2009.

Ergebnisse Die lithiumreiche Region der Atacama-Region zeigt eine signifikant geringere Suizidrate $(9,99$ pro 100.000$)$ als andere chilenische Landesteile $(12,50$ pro 100.000) ( $\mathrm{t}=4,75$; $\mathrm{df}=18, p<0,001)$.

Schlussfolgerungen Chilenische Regionen, die reich an natürlich vorkommenden Lithiumsalzen sind, zeigen niedrigere Suizidmortalitätsraten im Vergleich zu anderen Regionen. Obwohl auf Grund des Studiendesigns keine Kausalität nachgewiesen werden kann, bestärken diese Resultate frühere Studien und unterstreichen die Notwendigkeit weiterer Forschung über die Effekte natürlich vorkommenden, gering dosierten Lithiums auf die psychische Gesundheit.

Schlüsselwörter Lithium · Suizid · Chile · Lithiumvorkommen · Epidemiologie · Public Health · Soziale Faktoren

Impact of natural lithium ressources on suicide mortality in Chile 2000-2009: a geographical analysis

\section{Summary}

Background There is increasing evidence for the hypothesis that lithium salts at naturally occurring levels in drinking water may have a moderating effect on suicide rates of the exposed population. The aim of this study was to examine whether the lithium rich Atacama region in Chile is associated with lower suicide mortality in comparison to other regions.

Methods Suicide data was acquired from the Chilean Ministry of Health. Socio-economic variables (rate of unemployment, urbanity, median household income, percentage of indeginous population) were obtained for all regions of Chile from the national statistical institute. We calculated annual suicide rates per 100,000 
for each group for the years 2000-2009 and tested the hypothesis that suicide rates are lower in lithium rich regions in comparison to other regions of Chile.

Results The lithium rich Atacama Desert shows a significantly lower suicide rate $(9.99$ per 100,000$)$ in comparison to other parts of Chile $(12.50$ per 100,000$)(\mathrm{t}=$ 4.75, $\mathrm{df}=18, p<0.001$ ).

Conclusions Chilean regions rich in naturally occurring lithium salts show lower suicide mortality rates in comparison to other regions. Although causality cannot be proven by this design, these findings add to previous findings and warrant further research on the effects of naturally occurring low-dose lithium on health.

Keywords Lithium - Suicide - Chile · Environmental Lithium · Epidemiology · Public Health · Social Factors

\section{Einleitung}

Weltweit wird die Anzahl an Suiziden pro Jahr auf 1 Mio. geschätzt. Dies entspricht einer jährlichen Mortalitätsrate von ca. 15 pro 100.000 . Global gesehen ist Suizid mit bis zu 1,5\% aller Todesfälle die zehnthäufigste Todesursache [1] und gilt als die zweithäufigste Todesursache in der Altersgruppe zwischen 15 und 29 Jahren [2]. Chile hat mit 14,7 auf 100.000 Einwohner die höchste Suizidmortalitätsrate Südamerikas [3].

Umstritten ist, ob Lithiumsalzkonzentrationen in der Umwelt und die resultierende Aufnahme in den Organismus möglicherweise antisuizidale Eigenschaften besitzen. Zuletzt gab es mehrere Berichte über ein inverses Verhältnis zwischen Lithiumkonzentrationen im Trinkwasser und Suizidraten. Solche Ergebnisse wurden für Texas [4, 5], Japan [6], Österreich [7] und Griechenland [8] gezeigt. Eine Studie in OstEngland zeigte jedoch keine solche Assoziation [9]. Für Texas und Österreich blieb die Korrelation auch nach Einbeziehung sozioökonomischer Faktoren signifikant [5, 7].

Die nördlichen Regionen Chiles (Atacama-Wüste) weisen weltweit die höchste Konzentration an natürlich vorkommenden Lithiumsalzablagerungen [4, $10,11]$ und die höchste Lithiumsalzkonzentration in Oberflächengewässern [12] auf. Chile ist mit seinem zusammenhängenden Kutur- und Sprachraum mit gemeinsamer Juridikatur trotz ausgeprägter NordSüd-Ausdehnung und seinen nach einheitlichen Erhebungsmethoden durch ein nationales Statistikamt zentral gesammelten Suizidmortalitätsdaten eine interessante Region für Untersuchungen. Dies gilt insbesondere für die Epidemiologie der Suizidmortalität, aber auch im Hinblick auf die Untersuchung von Expositionseffekten durch natürliche Lithiumsalzvorkommen [12-16].

Der antisuizidale Effekt von Lithium, welches als Stimmungsstabilisator [17] seit mehr als 60 Jahren in klinischem Gebrauch ist, ist mehrfach dokumentiert $[18,19]$. Therapeutische Dosen für die Behandlung etwa der bipolaren Störung sind bedeutend höher als durchschnittliche Umweltkonzentrationen von Lithium. Die durchschnittliche Tagesdosis an gelöstem Lithium zur Etablierung von Serumkonzentrationen zwischen 0,5 bis 1,0 mmol/l, wie von der British Association for Psychopharmacology empfohlen, beträgt $70 \mathrm{mg}[20,21]$.

Natürliche Exposition gegenüber Lithium im Trinkwasser erreicht Größenordnungen zwischen $1 \mu \mathrm{g} / \mathrm{l}$ und $0,1 \mathrm{mg} / \mathrm{l}[10,22]$. In bestimmten chilenischen Oberflächengewässern kann die Lithiumkonzentration bis zu $207 \mathrm{mg} / \mathrm{l}$ (Muestra Salina, Sector Misitune) betragen, das entspricht dem 100- bis 10.000-fachen im Vergleich zu nordamerikanischen Flüssen [10]. Weiters wurde gezeigt, dass die Bevölkerung in lithiumreichen Regionen Chiles im Vergleich $\mathrm{zu}$ anderen Regionen weltweit eine hohe Lithiumzufuhr aus verschiedenen Nahrungsmitteln erhalten, in manchen Fällen äquivalent $\mathrm{zu}$ therapeutischen Dosen bei der Behandlung der bipolaren Störung [11].

Ziel dieser Studie war eine Abbildung der geographischen Verteilung und zeitlichen Veränderung der Suizidmortalitätsraten in Chile im Jahrzehnt von 2000 bis 2009. Als Näherungswert für die Exposition gegenüber Lithium in der Umwelt wurde, entsprechend der geographischen Verteilung der lithiumreichen Gebiete, ein fallender Lithiumspiegel mit zunehmendem Längengrad (Entfernung vom lithiumreichen Norden) postuliert. Unter der Voraussetzung, dass die Bevölkerung des nördlichen Chile durch Wasser- und Nahrungsquellen hohen Lithiumspiegeln in der Umwelt ausgesetzt sind [10, 11], war es unsere Hypothese, dass die Suizidraten in der Atacama-Region niedriger sein würden als im Rest Chiles.

\section{Datenerhebung und Methodik}

\section{Datenerhebung}

Suizidstatistiken von den Jahren 2000 bis 2009 wurden vom chilenischen Gesundheitsministerium erhoben. Daten wurden als Suizidrate und in absoluten Zahlen je Verwaltungsbezirk und Geschlecht angegeben. Quelle war die offizielle Suizidmortalitätsstatistik entsprechend der internationalen Klassifikation (ICD-10: X60-X84).

Sozioökonomische Variablen wurden nach Regionen und Geschlecht getrennt für alle chilenischen Regionen vom Nationalen Statistikinstitut Chiles (Instituto Nacional de Estadísticas [INE Chile]) angefordert. Diese stammen aus der Volkszählung aus dem Jahr 2002. Die regionalen Daten umfassten insbesondere solche Werte, die aus vorangegangenen Studien als mit der Suizidrate der betroffenen Bevölkerung assoziiert gelten: Der Anteil an indigener Bevölkerung [1], der Grad der Urbanisierung als Anteil der Bevölkerung in Städten nach INE-Definition [23, 24] und ökonomische Parameter [25] wie die Arbeitslosenrate und das mediane Einkommen pro Haushalt [26]. Medianes 


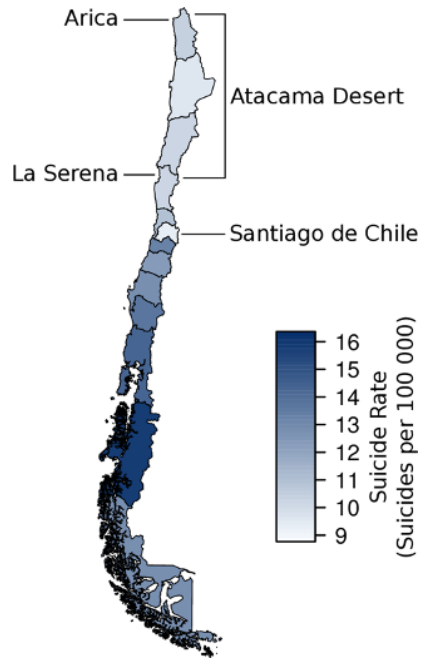

Abb. 1 Karte von Chile mit den Suizidraten, eingezeichnet pro 100.000Einwohnernje Regionfür die Jahre2000-2009. (Anmerkung: Die ausgeprägte dunkle Färbung vor der Südwestküste Chile entspricht der Schwarzumrandung der vorgelagerten Inseln)

Monatseinkommen wurde als Vielfaches von 1000 chilenischen Pesos (CLP) angegeben. Für die geographische Korrelation wurde für jede Region der Breitengrad (negativ vom Äquator aus südwärts gemessen) der Stadt mit der größten Population in der jeweiligen Region angegeben.

\section{Statistik}

Suizidraten (SR) wurden als Anzahl an Suiziden pro 100.000 Einwohner pro Jahr angegeben. Arbeitslosigkeit wurde als Anteil der uneingeschränkt arbeitsfähigen Personen ohne gegenwärtige Beschäftigung dividiert durch alle uneingeschränkt arbeitsfähigen Personen berechnet. Als allgemeine deskriptive Statistik wurde eine Querschnittsanalyse von Suizidraten und Altersstruktur in den Jahren 2000 bis 2009 angewandt. Die longitudinale Analyse konzentrierte sich auf den Verlauf der Suizidrate während der Periode von 2000 bis 2009, mit Einbeziehung der Urbanisierung. Wir prüften den Einfluss sozioökonomischer Faktoren und geographischer Breite auf die Suizidrate je nach Geschlecht und insgesamt mittels Rangsummentest und Spearman-Koeffizient $\rho$.

Die Haupthypothese (Suizidraten in den lithiumreichen Gegenden der Atacama sind niedriger als im Rest Chiles) wurde geprüft, indem Chile in zwei Gruppen geteilt wurde: „Atacama“ und „Nicht-Atacama“. Wir berechneten jährliche Suizidraten pro 100.000 für jede Gruppe in den Jahren 2000 bis 2009. Die Analysen wurden für beide Geschlechter und für die Gesamtbevölkerung getrennt durchgeführt. „Atacama" entspricht den nördlichen Regionen I-III vor dem Jahr 2007 und „Nicht-Atacama“ wurde als südliche Region definiert (Regionen IV-XII vor 2007). Die Großstadt Santiago de Chile wurde aus der Analyse ausgeschlossen (Nicht-Atacama), da sie, gemessen an Urbanisierung und Bevölkerungszahl, nicht vergleichbar mit der übrigen Region war. Sie war die einzige Region mit signifikantem Ausreißerwert in einem Skewness-adjusted multivariaten Ausreißertest, der Bevölkerungszahl, Suizidzahlen, Urbanisierung und Gesamteinkommen einbezog (out $_{\text {adj }}=1,0 \times 10^{16}>$ out $_{\text {crit }}=8,6 \times 10^{15}$ ) [27].

Die Robustheit der zugrundeliegenden Daten wurde dahingehend überprüft, dass nach einer Assoziierung des Ortes und der Suizidraten mit Moran's I zur Suche nach einer ortsbezogenen Autokorrelation [28] gesucht wurde. Es sollte ausgeschlossen werden, dass die Assoziationen mit den Suizidraten in den Regionen „Atacama“ oder „Nicht-Atacama“ auf Grund von anderen Parametern verursacht werden.

Alle statistischen Untersuchungen wurden mit Hilfe der R Software (Version 3.0.1 für x86_64 pc-linuxgnu) durchgeführt. Das Kartenmaterial entstammt dem GADMv2 Datenset mit einer Erweiterung um die Atacama Wüste nach der Definition der WWF eco regions $[28,29]$ und wurde mit Quantum GIS 1.8 unter GNU/Linux für die Benutzung mit $\mathrm{R}$ spatial libraries aufbereitet.

\section{Resultate}

Die mittlere Suizidrate (pro 100.000) in der Zeit von 2000 bis 2009 in Chile betrug 11,0 für beide Geschlechter - 18,5 für Männer und 3,7 für Frauen. Diese Raten ergeben ein Verhältnis von 5,1 zu 1 zwischen Männern und Frauen.

In der untersuchten Zeitspanne von zehn Jahren stiegen die Suizidraten sowohl für Männer (von 16,6 pro 100.000 in 2000 auf 20,6 in 2009, Spearman-Koeffizient $\rho=0,84, p=0,004$ ) und Frauen (von 2,7 in 2000 auf 5,0 in 2009, $\rho=0,99, p<0,001$ ) als auch insgesamt (von 9,6 in 2000 auf 12,7 in 2009, $\rho=0,87, p=0,003$ ).

Weiters untersuchten wir die zeitliche Veränderung in der Großstadtregion Santiago de Chile und in Chile exklusive der Großstadtregion Santiago de Chile separat, da $33,7 \%$ aller Suizide in Santiago stattfanden und die Region als einzige Metropole sich in zahlreichen Charakteristika von den übrigen Regionen unterscheidet. Chile als auch Santiago de Chile wiesen einen signifikanten Suizidratenanstieg während des untersuchten Zeitraums auf (alle Zeit-Analysen zeigen einen Spearman-Koeffizienten $\rho>0,80, p<0,01$ ).

\section{Geographische Verteilung}

Wie in Abb. 1 und Tab. 1 dargestellt, zeigen die Suizidraten in Chile eine inhomogene geographische Verteilung. Diese Heterogenität ist auch durch eine signifikant positive räumliche Autokorrelation der absoluten Suizidraten pro Region mit der räumlich korrigierten Suizidrate (Moran's I =0,59, $p=0,004$ ) gekennzeichnet. Das geographische Muster der Suizidraten in Chile blieb während der untersuchten Zeitspan- 
Tab. 1 Jährliche Suizidraten und Populationsgröße je Region von 2000 bis 2009

\begin{tabular}{|l|l|l|l|l|l|}
\hline & Region & Suizidrate gesamt & Suizidrate Männer & Suizidrate Frauen & Population \\
\hline I & Tarapacá & 10,3 & 17,0 & 3,4 & $466.321,8$ \\
\hline II & Antofagasta & 9,7 & 15,2 & 3,7 & $537.345,2$ \\
\hline III & Atacama & 10,2 & 17,5 & 2,7 & $269.353,9$ \\
\hline IV & Coquimbo & 10,2 & 17,1 & 3,3 & $661.716,9$ \\
\hline V & Valparaíso & 10,9 & 18,1 & $1.651 .911,9$ \\
\hline RM & Región Metropolitana & 9,2 & 15,3 & 3,9 & $6.497 .526,4$ \\
\hline VI & Lib. B. O'Higgins & 13,2 & 22,4 & $835.720,8$ \\
\hline VII & Maule & 12,3 & 20,8 & $962.583,8$ \\
\hline VIII & Bío-Bío & 12,7 & 21,7 & $1.960 .802,6$ \\
\hline IX & Araucanía & 13,7 & 24,3 & $924.401,5$ \\
\hline X & Lagos & 14,3 & 24,1 & $1.149 .690,4$ \\
\hline XI & Aisén & 15,9 & 27,1 & $98.750,7$ \\
\hline XII & Magallanes y Antártica & 12,8 & 3,2 & $155.701,3$ \\
\hline- & Chile & 11,0 & 20,0 & $16.171 .827,2$ \\
\hline Alle Suizidraten pro 100.000 Einwohner. Die Population ist angegeben als die mittlere jährliche Population in den Jahren 2000 bis 2009. Die Regionen sind nach \\
\hline ihrer geographischen Lage von Norden (I) nach Süden (XII) gelistet.
\end{tabular}

ne (2000-2009) vergleichsweise stabil, veranschaulicht durch die Korrelation der regionalen Suizidraten der Jahre 2000 und 2009 (Spearman-Rangsummentest $\rho=$ $0,71, p=0,009)$.

Die Spearman-Rangsummenkorrelation der absoluten Suizidraten mit geographischer Breite war hoch ( $\rho=-0,80, p=0,002$ ). Bei Männern hatten die nördlichsten Regionen und die Großstadtregion Santiago die niedrigsten Suizidraten (15,1-17,1 pro 100.000), stiegen Richtung Süden stetig bis auf 27,1 pro 100.000 in der Region Aisén an. Suizidraten für Frauen zeigten ein ähnliches Muster mit der niedrigsten Suizidrate in der Atacama-Region (2,7 pro 100.000) und der höchsten in der südlichen Region Magallanes und Antártica Chilena mit 4,9 pro 100.000 (Tab. 1).

\section{Prüfung der Atacama-Hypothese}

Chile wurde in einen nördlichen Teil, der AtacamaRegion entsprechend, und in einen südlichen Teil geteilt (Abb. 1). Die berechneten Suizidraten pro Jahr für die Jahre von 2000 bis 2009 wurden für jeden
Teil verglichen. Suizidraten zeigten einen signifikanten Unterschied zwischen der Atacama-Region (9,99 pro 100.000) und Nicht-Atacama-Region (12,50 pro 100.000) $(\mathrm{t}=4,75$, $\mathrm{df}=18, p<0,001)$. Dem NordSüd-Gefälle und damit zunehmender Entfernung von Atacama entsprechend war geographische Breite mit der Suizidrate der Gesamtbevölkerung $(\rho=-0,80, p=$ $0,001)$ und in der Gruppe der Männer $(\rho=-0,79, p=$ $0,001)$ signifikant korrelierend und zeigte einen Trend zur Signifikanz unter Frauen $(\rho=-0,50, p=0,082)$.

Nach Miteinbeziehung des Ausreißers Santiago de Chile zeigte der Suizidratenunterschied zwischen Norden und Süden noch einen Trend zur Signifikanz (9,99 versus 11,$06 ; \mathrm{t}=2,07, \mathrm{df}=18, p=0,053$ ).

\section{Überprüfung anderer sozioökonomischer Variablen}

Medianes Pro-Kopf-Einkommen, Arbeitslosigkeit und indigener Bevölkerungsanteil korrelierten in unserer Untersuchung nicht signifikant mit Suizidraten. Urbanisierung jedoch war signifikant negativ korreliert mit Suizidraten insgesamt ( $\rho=-0,63, p=0,022)$ (Tab. 2).

Tab. 2 Bivariate Spearman Rangsummentest Korrelation $\rho$ von verschiedenen möglichen Einflussfaktoren

\begin{tabular}{|c|c|c|c|c|c|c|c|c|}
\hline & \multirow[b]{2}{*}{ Variable } & \multicolumn{7}{|c|}{ Variable (siehe Spalte) } \\
\hline & & (1) & (2) & (3) & (4) & (5) & (6) & (7) \\
\hline (1) & $\begin{array}{l}\text { Gesamt Suizidrate pro } \\
100.000\end{array}$ & - & $-0,30$ & $-0,21$ & 0,41 & $-0,63^{*}$ & $0,64^{*}$ & $-0,80^{\star \star}$ \\
\hline (2) & Medianes Einkommen & $-0,30$ & - & $-0,52$ & 0,21 & $0,81^{\star \star \star}$ & $-0,43$ & 0,15 \\
\hline (3) & Arbeitslosigkeit & $-0,21$ & $-0,52$ & - & $-0,23$ & $-0,35$ & 0,03 & 0,44 \\
\hline (4) & $\begin{array}{l}\text { Anteil indigener Bevöl- } \\
\text { kerung }\end{array}$ & 0,41 & 0,21 & $-0,23$ & - & 0,13 & $-0,16$ & $-0,29$ \\
\hline (5) & \% Bevölkerung in Stadt & $-0,63^{*}$ & $0,81^{\star * *}$ & $-0,35$ & 0,13 & - & $-0,80^{\star * \star}$ & 0,47 \\
\hline (6) & $\begin{array}{l}\text { Veränderung in der \% } \\
\text { der Bevölkerung in der } \\
\text { Stadt }\end{array}$ & $0,64^{*}$ & $-0,43$ & 0,03 & $-0,16$ & $-0,80^{\star \star \star}$ & - & $-0.61^{*}$ \\
\hline (7) & Breitengrad & $-0.80^{* *}$ & 0.15 & 0.44 & -0.29 & 0.47 & -0.61 & - \\
\hline
\end{tabular}




\section{Diskussion}

Unsere Resultate zeigen, dass die lithiumreiche Atacama-Region in Chile signifikant niedrigere Suizidraten aufweist als der Rest Chiles. Die Resultate stimmen mit zuletzt zunehmenden ökologischen Studien aus Texas [4, 5], Japan [6, 30], Österreich [7] und Griechenland [8] überein, die nachweisen konnten, dass Lithiumvorkommen in der Umwelt - speziell im Trinkwasser - mit einer niedrigeren Suizidmortalität assoziiert sind.

Obwohl wir selbst keine direkten Messungen von Lithium im chilenischen Trinkwasser vorgenommen hatten, ist es ein anerkannter Umstand, dass die nördlichen Regionen Chiles (Atacama-Wüste) reich an natürlichen Lithiumablagerungen sind $[4,10,11]$, mit hohen Lithiumkonzentrationen in Oberflächengewässern bis zu $207 \mathrm{mg} / \mathrm{l}$ [10, 12]. Diese Werte sind signifikant höher als in anderen Ländern, in denen die Lithiumhypothese getestet wurde - wie in Texas (bis zu 0,219 mg/l) oder Österreich (bis zu 1,3 mg/l) [5, 7]. Es wurde gezeigt, dass die den in der Umwelt vorkommenden Lithiumsalzen ausgesetzte Bevölkerung Chiles eine bedeutend höhere Lithiumzufuhr im Vergleich zur Bevölkerung in anderen Regionen aufweist. Teilweise werden sogar klinisch therapeutische Dosen erreicht [11]. Indem wir die geographische Breite als Ersatzparameter für Lithiumexposition durch die Umwelt einsetzten, konnten wir zeigen, dass die Suizidraten mit zunehmender Entfernung von der AtacamaRegion steigen.

Der zugrundeliegende Mechanismus der Assoziation zwischen Suizidraten und geographischer Breite - wie in unserer Studie gezeigt - wurde häufig mit Unterschieden in der Sonnenlichtexposition begründet. Frühere Studien aus Chile, die gezeigt haben, dass die saisonale Variabilität an Suizidinzidenz in Regionen mit zunehmender geographischer Breite - und daher auch weiter entfernt von Atacama - größer ist [16], stimmen mit der Literatur über Zusammenhänge zwischen Sonnenlicht und Suizidmortalität überein [31]. So ändert sich der Einfallswinkel und die Dauer der Insolation pro Tag je nach Breitengrad und nimmt mit der Entfernung vom Äquator ab. Weiter differenziert wird diese Assoziation unter anderem von Vyssoki et al. [32] und von Tsai und Cho [33] erörtert. Diese berichten von signifikanten Korrelationen zwischen Sonnenlicht beziehungsweise Wärmestrahlung und Suizidmortalität.

Andererseits sind die Resultate auch passend $\mathrm{zu}$ unserer in diesem Artikel vorgestellten Hypothese. Es scheint plausibel, dass die niedrigere saisonale Variabilität der Suizidmortalität im Norden Chiles auch dem bekannten stimmungsstabilisierenden und antisuizidalen Effekt von Lithium geschuldet sein könnte [16]. Allerdings muss in Betracht gezogen werden, dass die Amplituden suizidaler Saisonalität in Regionen mit niedrigen Suizidraten aus statistischen Gründen eher gering sind - wie schon in der Literatur beschrieben [34].

Der Wirkungsmechanismus der postulierten suizidprotektiven Eigenschaften von Lithium, insbesondere in niedrigen Dosierungen, ist noch ungeklärt $[35,36]$. Es gibt Nachweise, dass Lithium das Volumen von zerebralen Strukturen mit Beteiligung an emotionaler Steuerung wie dem präfrontalen Kortex, dem Hypocampus und der Amygdala vor Abbau bewahrt. Auf molekularer Ebene wirkt Lithium auf Neurotransmitter-modulierende Second-MessengerSysteme wie die Adenylatcyclase und Proteinkinase $C$ und dämpft dadurch eventuell Überschuss an Neurotransmission und oxidativem Stress. Zusätzlich wurde gezeigt, dass Lithium die Expression neuroprotektiver Proteine wie Brain-derived neurotrophic factor steigert, Glykogensynthase-Kinase 3 inhibiert und dadurch apoptotische Prozesse reduziert bzw. Neurogenese stimuliert [35, 36]. Weitere Studien zur Auswirkung von niedrig dosiertem Lithium auf das zentrale Nervensystem scheinen gerechtfertigt.

Unsere Resultate zeigen auch eine inverse Assoziation zwischen Urbanisierung und Suizidraten: Ländliche Regionen weisen eine höhere Suizidmortalität auf. Dieses Ergebnis steht im Einklang zu Resultaten anderer Studien. Diese bieten für das Land-Stadt-Gefälle von Suizidraten mögliche Erklärungsmodelle, wie größere Barrieren bis zur Inanspruchnahme von psychiatrischen oder psychotherapeutischen Einrichtungen (Verfügbarkeit und Erreichbarkeit), ländliche Kultur, Einstellungen der Gesellschaft zu und Stigmatisierung von psychischen Erkrankungen und Hilfsbedürftigkeit sowie die Exposition gegenüber und Verfügbarkeit von Suizidhilfsmitteln wie Waffen und Giften [37]. Traditionell waren weltweit Suizidraten in ländlichen Regionen niedriger als solche in Städten. In manchen Ländern hat sich dieser Umstand über die letzten Jahrzehnte hinweg jedoch gewandelt. Interessanterweise zeigten unsere Resultate keine signifikante Verbindung zwischen indigenem Bevölkerungsanteil und den Suizidraten der betroffenen Region. Für die indigene Bevölkerung südamerikanischer Länder wie Brasilien wurden bisher höhere Suizidraten berichtet (12,6 pro 100.000 im Gegensatz zu 5,3 für die übrige Bevölkerung in den Jahren 2006 bis 2010) [38]. Es kann zum jetzigen Zeitpunkt nur darüber spekuliert werden, warum sich in Chile dieser Umstand in den Ergebnissen nicht dargestellt hat.

\section{Limitationen}

Die Unterscheidung zwischen lithiumexponierter und nicht-lithiumexponierter Umwelt basiert auf früheren Daten. Eigene Messungen an zuvor spezifizierten geographischen Koordinaten, aus denen ein Modell der geographischen Verteilung erstellt und mit einem Verteilungsmodell von Suizidraten korreliert werden könnte, würden eine detailliertere und umfassendere Analyse ermöglichen. 
Ebenfalls limitierend ist das fehlende Ausmaß an Detailliertheit der Daten in der nationalen Suiziddatenbank. Es sind Fallzahlen pro Gesundheitsorganisationsbezirk (kongruent mit politischen Grenzen) pro Jahr und Geschlecht angegeben. Daten aus kleineren geographischen Organisationseinheiten sowie detailliertere Daten zu den jeweiligen Suiziden würden das statistische Modell verbessern, waren aber vom INE Chile nicht verfügbar.

\section{Implikationen}

Unsere Resultate unterstützen die zunehmende Datenlage für eine inverse Beziehung zwischen Lithiumkonzentrationen in der Umwelt und Suizidmortalität. Sind die Ergebnisse dieser ökologischen Studie zwar interessant und übereinstimmend mit früheren Untersuchungen, so müssten sie doch erst mittels zukünftiger prospektiver Studien und explorativer Forschung bestätigt werden. Die von manchen Autoren vorgebrachte Überlegung, Trinkwasser zur Reduktion von Suizidraten in Hochrisiko-Bevölkerungen mit Lithium zu versetzen, wäre allerdings verfrüht und wird von uns keinesfalls unterstützt. Es besteht Bedarf genau abzuwägender Sicherheitsüberlegungen und weiterer Forschung $\mathrm{zu}$ diesem Thema. Ebenso scheint es uns wichtig, zur Forschung über indigene Bevölkerungsgruppen Chiles anzuregen, da wir in unseren Daten, anders als es bisher gezeigt werden konnte, keinen Zusammenhang zwischen dem Anteil der indigenen Bevölkerung und den Suizidmortalitätsraten finden konnten. Ob dieses Ergebnis in künftigen Studien und entsprechenden Studiendesigns repliziert werden kann, bleibt abzuwarten.

Danksagung Wir möchten uns bei Dr. Gary Giulian von der Abteilung für Biomolekulare Messungen am nationalen Institut für Standards und Technologie der Vereinigten Staaten von Amerika für seine wertvollen Anmerkungen und Hinweise zu unserem Projekt bedanken. Ebenso danken wir Dr. Jakob Klein für die Mitarbeit an früheren Versionen des Manuskripts, Programmierung in R und für erste statistische Analysen.

Förderung Diese Forschung wurde unterstützt durch die Medizinische Universität Wien, welche als Arbeitsgeber von Daniel König, Nicole Baus, Victor Blüml, Josef Baumgartner und Nestor D. Kapusta fungiert. Der korrespondierende Autor hatte vollen Zugriff auf alle Daten und ist verantwortlich für die endgültige Entscheidung zur Publikation.

Open access funding provided by Medical University of Vienna.

\section{Einhaltung ethischer Richtlinien}

Interessenkonflikt D. König, J. Baumgartner, V. Blüml, A. Heerlein, C. Téllez, N. Baus und N.D. Kapusta geben an, dass kein Interessenkonflikt besteht.

Ethische Standards Da keine Daten von Individuen erhoben wurden und das zugrundeliegende Material von staatlichen
Institutionen erhoben und zugänglich gemacht wurde, war kein Votum einer Ethikkomission notwendig.

Open Access Dieser Artikel wird unter der Creative Commons Namensnennung 4.0 International Lizenz (http:// creativecommons.org/licenses/by/4.0/deed.de) veröffentlicht, welche die Nutzung, Vervielfältigung, Bearbeitung, Verbreitung und Wiedergabe in jeglichem Medium und Format erlaubt, sofern Sie den/die ursprünglichen Autor(en) und die Quelle ordnungsgemäß nennen, einen Link zur Creative Commons Lizenz beifügen und angeben, ob Änderungen vorgenommen wurden.

\section{Literatur}

1. Hawton K, van Heeringen K. Suicide. Lancet. 2009;373 (9672):1372-81.doi:10.1016/s0140-6736(09)60372-x.

2. Weltgesundheitsorganisation. Preventing Suicide: A Global Imperative. World Health Organization 2014. http:// www.who.int/mental_health/suicide-prevention/exe_ summary_english.pdf?ua=1.Zugegriffen:27. Nov.2014.

3. Bustamante F, Ramirez V, Urquidi C, Bustos V, Yaseen Z, Galynker I. Trends and Most Frequent Methods of Suicide in Chile Between 2001 and 2010. Crisis. 2016;37(1):21-30. doi:10.1027/0227-5910/a000357.

4. Schrauzer GN, Shrestha KP. Lithium in drinking water and the incidences of crimes, suicides, and arrests related to drug addictions. Biol TraceElem Res. 1990;25(2):105-13.

5. Bluml V, Regier MD, Hlavin G, Rockett IR, Konig F, Vyssoki B, Bschor T, Kapusta ND. Lithium in the public water supply and suicide mortality in Texas. J Psychiatr Res. 2013;47(3):407-11. doi:10.1016/j.jpsychires.2012.12.002.

6. Ohgami H, Terao T, Shiotsuki I, Ishii N, Iwata N. Lithium levels in drinking water and risk of suicide. The British journal of psychiatry. J Ment Sci. 2009;194(5):464-5, discussion 446 doi:10.1192/bjp.bp.108.055798.

7. Kapusta ND, Mossaheb N, Etzersdorfer E, Hlavin G, Thau K, Willeit M, Praschak-Rieder N, Sonneck G, Leithner-Dziubas $\mathrm{K}$. Lithium in drinking water and suicide mortality. The Britishjournal of psychiatry. J MentSci. 2011;198(5):346-50. doi:10.1192/bjp.bp.110.091041.

8. Giotakos O, Nisianakis P, Tsouvelas G, Giakalou VV. Lithium in the public water supply and suicide mortality in Greece. Biol Trace Elem Res. 2013;156(1-3):376-9. doi:10.1007/ s12011-013-9815-4.

9. Kabacs N, Memon A, Obinwa T, Stochl J, Perez J. Lithium in drinking water and suicide rates across the East of England. The British journal of psychiatry. J Ment Sci. 2011;198(5):406-7.doi:10.1192/bjp.bp.110.088617.

10. Figueroa L, Barton S, Schull W, Razmilic B, Zumaeta O, Young A, Kamiya Y, Hoskins J, Ilgren E. Environmental lithium exposure in the North of Chile - I. Natural water sources. Biol Trace Elem Res. 2012;149(2):280-90. doi:10. 1007/s12011-012-9417-6.

11. Figueroa LT, Razmillic B, Zumeata O, Aranda GN, BartonSA, Schull WJ, Young AH, Kamiya YM, Hoskins JA, Ilgren EB. Environmental lithium exposure in the north of Chile-II. Natural food sources. Biol Trace Elem Res. 2013;151(1):122-31. doi:10.1007/s12011-012-9543-1.

12. Figueroa LT, Barton SA, Schull W, Young AH, Kamiya YM, Hoskins JA, Ilgren EB. Environmental lithium exposure in the north of Chile - Tissue exposure indices. Epidemiol Biostat Public Health. 2014;11:1.doi:10.2427/8847.

13. Harari F, Langeen M, Casimiro E, Bottai M, Palm B, Nordqvist $H$, Vahter M. Environmental exposure to lithium during pregnancy and fetal size: A longitudinal study in 
theArgentinean Andes. Environ Int.2015;77c:48-54.doi:10. 1016/j.envint.2015.01.011.

14. Lake IR, Foxall CD, Fernandes A, Lewis M, Rose M, White O, Lovett AA, White S, Dowding A, Mortimer D. The effects of flooding on dioxin and PCB levels in food produced on industrial river catchments. Environ Int. 2015;77c:106-15. doi:10.1016/j.envint.2015.01.006.

15. Munoz-Quezada MT, Iglesias V, Lucero B, Steenland K, Barr DB, Levy K, Ryan PB, Alvarado S, Concha C. Predictors of exposure to organophosphate pesticides in schoolchildren in the Province of Talca. Chile Environ Int. 2012;47:28-36. doi:10.1016/j.envint.2012.06.002.

16. Heerlein A, Valeria C, Medina B. Seasonal variation in suicidal deaths in Chile: its relationship to latitude. Psychopathology.2006;39(2):75-9. doi:10.1159/000090596.

17. Altamura AC, Lietti L, Dobrea C, Benatti B, Arici C, Dell'Osso B. Mood stabilizers for patients with bipolar disorder: the state of the art. Expert Rev Neurother. 2011;11(1):85-99. doi:10.1586/ern.10.181.

18. Cipriani A, Hawton K, Stockton S, Geddes JR. Lithium in the prevention of suicide in mood disorders: updated systematic review and meta-analysis. BMJ. Clin Res Ed. 2013;3646:346. doi:10.1136/bmj.f3646.

19. Muller-Oerlinghausen B, Lewitzka U. Lithium reduces pathological aggression and suicidality: a mini-review. Neuropsychobiology. 2010;62(1):43-9. doi:10.1159/000314309.

20. Goodwin GM (2009) Evidence-based guidelines for treating bipolar disorder: revised second edition - recommendations from the British Association for Psychopharmacology. Journal of psychopharmacology (Oxford, England) 23(4):346-388. doi:10.1177/0269881109102919

21. Kapusta ND, Konig D. Naturally occurring low-dose lithium in drinking water. J Clin Psychiatry. 2015;76(3):e373-374. doi:10.4088/JCP.14com09574.

22. Schrauzer GN. Lithium: occurrence, dietary intakes, nutritional essentiality. J Am Coll Nutr. 2002;21(1):14-21. doi:10. 1080/07315724.2002.10719188.

23. Kapusta ND, Zorman A, Etzersdorfer E, Ponocny-Seliger E, Jandl-Jager E, Sonneck G. Rural-urban differences in Austrian suicides. Soc Psychiatry Psychiatr Epidemiol. 2008;43(4):311-8. doi:10.1007/s00127-008-0317-1.

24. INE. Población y Sociedad: Aspectos Demográficos. Publicación Especial: Instituto Nacional de Estadísticas; 2008.

25. Rehkopf DH, Buka SL. The association between suicide and the socio-economic characteristics of geographical areas: a systematic review. Psychol Med. 2006;36(2):145-57. doi:10.1017/s003329170500588x.

26. INE. Ingreso de Hogares y Personas: Encuesta Suplementaria de Ingresos: Instituto Nacional de Estadísticas;; 2003.

27. Byrs G, Hubert M, Rousseeuw PJ. A Robustification of Independent Component Analysis. J Chemom. 2005;19:346-75.

28. Moran PA. The interpretation of statistical maps. JR Stat Soc Ser B. 1948;10(2):243-51.

29. WWF Western South America: Northwestern Chile. World Wildlife Fund. http://www.worldwildlife.org/ecoregions/ nt1303.Zugegriffen:26. Juni 2013.

30. Sugawara N, Yasui-Furukori N, Ishii N, Iwata N, Terao T. Lithium in tap water and suicide mortality in Japan. Int J Environ Res Public Health. 2013;10(11):6044-8. doi:10. 3390/ijerph10116044.

31. Preti A. The influence of climate on suicidal behaviour in Italy. Psychiatry Res. 1998;78(1-2):9-19.

32. Vyssoki B, Kapusta ND, Praschak-Rieder N, Dorffner G, Willeit M. Direct effect of sunshine on suicide. JAMA Psychiatry. 2014;71(11):1231-7. doi:10.1001/jamapsychiatry.2014. 1198.

33. Tsai JF, Cho W. Temperature change dominates the suicidal seasonality in Taiwan: a time-series analysis. JAffect Disord. 2012;136(3):412-8. doi:10.1016/j.jad.2011.11.010.

34. Nader IW, Pietschnig J, Niederkrotenthaler T, Kapusta ND, Sonneck G, Voracek M. Suicide seasonality: complex demodulation as a novel approach in epidemiologic analysis. PLOS ONE. 2011;6(2):e17413. doi:10.1371/journal.pone. 0017413

35. Malhi GS, Tanious M, Das P, Coulston CM, Berk M. Potential mechanisms of action of lithium in bipolar disorder. Current understanding. CNS Drugs. 2013;27(2):135-53. doi:10. 1007/s40263-013-0039-0.

36. Quiroz JA, Machado-Vieira R, Zarate CA Jr., Manji HK. Novel insights into lithium's mechanism of action: neurotrophic and neuroprotective effects. Neuropsychobiology. 2010;62(1):50-60. doi:10.1159/000314310.

37. Judd F, Cooper AM, Fraser C, Davis J. Rural suicide - people or place effects? Aust N Z J Psychiatry. 2006;40(3):208-16. doi:10.1111/j.1440-1614.2006.01776.x.

38. Souza ML, Orellana JD. Suicide among the indigenous people in Brazil: a hidden public health issue. Revista brasileira de psiquiatria (Sao Paulo, Brazil : 1999) 34 (4). 2012, S. 489-92. 